\title{
COMMUNICATIONS
}

\section{Topochemical photodimerization of 7-acetoxycoumarin: the acetoxy group as a steering agent}

\author{
N. Ramasubbu, K. Gnanaguru, K. Venkatesan, and V. Ramamurthy ${ }^{1}$ \\ Department of Organic Chemistry, Indian Institute of Science, Bangalore 560012, India
}

Received March 19, 1982

\begin{abstract}
N. Ramasubbu, K. Gnanaguru, K. Venkatesan, and V. Ramamurthy. Can. J. Chem. 60, 2159 (1982).
Among the various substituted coumarins investigated only 7-acetoxycoumarin is observed to photodimerize topochemically in the crystalline state and this observation may be of importance in the context of "crystal engineering".
\end{abstract}

N. Ramasubbu, K. Gnanaguru, K. Venkatesan et V. Ramamurthy. Can. J. Chem. 60, 2159 (1982).

Des diverses coumarines substituées étudiées, seule l'acétoxy-7 cou marine se photodimérise topochimiquement à l'état cristallin; cette observation peut avoir de l'importance dans le contexte de "l'ingénierie des cristaux".

[Traduit par le journal]

The photochemical and photophysical processes of many organic compounds are a function of the environment in which they are present. In this connection, we have chosen to investigate the environmental perturbations on the photodimerization of coumarins and the results of our study in the crystalline state are presented here. Coumarins have been chosen for our investigation as their fascinating photochemical behaviour has been fairly well understood (1) and therefore the environmental influence, which is the subject of our concern, would more easily be understandable. The type of bimolecular photochemical process which has been investigated extensively in the solid state is $\left[\pi_{2 s}+\pi_{2 s}\right]$ cycloaddition (2). It has been established that the success of this process in the solid state requires a packing arrangement in which the reacting double bonds are parallel to one another with mean interplanar distances $<$ ca. 4.1 $\AA$. The utility of such photoreactions as a synthetic tool is limited by the difficulty of achieving the desired type of crystal structure in any given case, for the factors that control the crystal packing are not fully understood. However, attempts to design molecules wherein the potentially reactive chromophores are brought into favourable orientation have been reported. So far as dimerization of olefins is concerned, the utilization of dichlorophenyl substituents has been of great value (3). A similar strategy, involving the strong tendency for oxygen atoms of ester groups to pack over the centre of the benzene ring of a neighbouring

'Author to whom correspondence may be addressed. molecule, has been utilized to "engineer" crystals (4). Scope undoubtedly exists for "engineering" many other crystals and the results presented below are important in this context.

Coumarin is known to be photostable in the solid state (5) although it readily dimerizes in solution (1). ${ }^{2}$ Therefore, this appeared to be a good molecular framework to study the role of substituents in bringing about the preferred molecular packing for photodimerization. Of the several coumarins examined by us only a few underwent photodimerization in the solid state upon uv irradiation ( $450 \mathrm{~W}$ medium pressure mercury lamp) as illustrated in Table 1. X-ray crystallographic studies of the monomers of 7-methoxy, 8-methoxy, and 4-chlorocoumarins completed in our laboratory reveal that packing arrangements in these crystals do not allow favorable orientation of the potentially reactive double bonds for dimerization to occur. These appear to be defect-initiated dimerizations (6). In this communication, we present our results on the solid state photodimerization of 7-acetoxycoumarin which is interesting in the context of crystal engineering. The mechanism of dimerization and the nature of the dimer are yet to be characterized in the case of other coumarins listed in Table 1.

The dimer $\left(\mathrm{mp} 215-217^{\circ} \mathrm{C}\right)$ formed in quantitative yield upon irradiation of 7-acetoxycoumarin

${ }^{2}$ Although coumarin is established to be photostable in the solid state, its unstable modification and its complex with mercuric chloride give syn head-head dimers in the solid state upon uv exposure. 
TABLE 1. Photoreactivity of coumarins in the solid state

\begin{tabular}{|c|c|c|c|c|}
\hline $\begin{array}{l}\text { S1. } \\
\text { No. }\end{array}$ & Compound & $\begin{array}{l}\text { Solvent of } \\
\text { crystallization }\end{array}$ & $\begin{array}{l}\text { Dimerization } \\
\text { solid state } \\
(\text { yield })^{a}\end{array}$ & $\begin{array}{l}\text { Nature of } \\
\text { the dimer }\end{array}$ \\
\hline 1. & Coumarin & Ethanol & No & $\ldots$ \\
\hline 2. & 4-Hydroxycoumarin & Water & No & - \\
\hline 3. & 4-Methoxycoumarin & Benzene & No & - \\
\hline 4. & 4-Acetoxycoumarin & Ethanol & No & - \\
\hline 5. & 4-Chlorocoumarin & Ethanol & Yes $(40 \%)$ & $\begin{array}{l}\text { syn Head-tail } \\
\text { anti Head-tail }\end{array}$ \\
\hline 6. & 7-Hydroxycoumarin & Ethanol & No & - \\
\hline 7. & 7-Methoxycoumarin & Benzene & $\begin{array}{c}\text { Yes } \\
(\sim 90 \%)\end{array}$ & syn Head-tail \\
\hline 8. & 7-Acetoxycoumarin & Chloroform & $\begin{array}{l}\text { Yes } \\
(\sim 90 \%)\end{array}$ & syn Head-head \\
\hline 9. & 7-Chlorocoumarin & Ethanol & Yes & $b$ \\
\hline 10. & 7-Methylcoumarin & Ethanol-water & Yes & $b$ \\
\hline 11. & 6-Chlorocoumarin & Ethanol & Yes & $b$ \\
\hline 12. & 6-Methoxycoumarin & Water & Yes & $b$ \\
\hline 13. & 8-Methoxycoumarin & Benzene & $\begin{array}{c}\text { Yes } \\
(\sim 50 \%)\end{array}$ & anti Head-tail \\
\hline 14. & 4-Methyl-7-hydroxycoumarin & Ethanol & No & - \\
\hline 15. & 4-Methyl-7-methoxycoumarin & Benzene & No & - \\
\hline 16. & 4-Methyl-7-chlorocoumarin & $\begin{array}{l}\text { Chloroform - } \\
\text { carbon tetrachloride }\end{array}$ & Yes & $b$ \\
\hline 17. & 4-Methyl-7-acetoxycoumarin & Acetone & Yes & $b$ \\
\hline
\end{tabular}

arradiated using $450 \mathrm{~W}$ medium pressure mercury lamp; yields were measured by nmr integration.

${ }^{D}$ Nature of the dimer is yet to be characterized.

was shown to have the $s y n$ head-head configuration based on its spectral properties: ir (Nujol): 1750 $\mathrm{cm}^{-1} ;{ }^{1} \mathrm{Hmr}$ (DMSO- $\left.d_{6}\right) \delta: 2.21(3 \mathrm{H}, \mathrm{s}), 4.22(2 \mathrm{H}$, $\mathrm{m}, \mathrm{AA}^{\prime} \mathrm{BB}^{\prime}$ pattern $), 6.66(1 \mathrm{H}, \mathrm{d}, J=2 \mathrm{cps}), 6.73$ $(1 \mathrm{H}, \mathrm{d} \times \mathrm{d}, J=2$ and $8.5 \mathrm{cps})$ and $6.92(1 \mathrm{H}, \mathrm{d}, J=$ $8.5 \mathrm{cps}) ; \mathrm{ms}\left(\mathrm{CI}, \mathrm{NH}_{3}\right): 426\left(\mathrm{M}+\mathrm{NH}_{4}{ }^{+}\right)$. Comparison of the above $\mathrm{nmr}$ spectral data with those published for the anti head-head dimer of 7 -acetoxycoumarin and the syn head-head dimers of polymethylene-dicarboxylic acid (7-coumarinyl) diesters (7) revealed that the above dimer is syn head-head.

In order to elucidate the nature of the intermolecular packing which leads to the formation of the observed dimer, a crystal structure analysis of 7-acetoxycoumarin was carried out. ${ }^{3}$ The packing thus obtained is displayed in Fig. 1.

${ }^{37}$-Acetoxycoumarin crystallizes from chloroform solution in the space group $P 2, / c$ with $a=3.833(2), b=22.665(4), c=$ $10.975(3) \AA, \beta=96.27(2)^{\circ}$, and $Z=4$. A total of 1789 reflections were collected on an Enraf Nonius CAD-4 diffractometer $(\lambda=$ $1.5418 \AA, \omega / 2 \theta$ scan) out of which 1016 were unique and significant $\left[\left|F_{\mathrm{o}}\right|>3 \sigma\left(\left|F_{\mathrm{o}}\right|\right)\right]$. The direct methods programme package MULTAN-80 was used for the structure solution (9). Full matrix least-squares refinement of positional and anisotropic thermal parameters of non-hydrogen atoms (treating hydrogen atoms isotropically) led to an $R$ value of 0.056 . The computer data on which this calculation is based are available, at a nominal charge, from the Depository of Unpublished Data, CISTI, National Research Council of Canada, Ottawa, Ont., Canada K1A 0S2.
The 7-acetoxycoumarin possesses so-called $\beta$-type packing which is characterized by the close packing of parallel and nearly planar reactive double bonds (8) with a separation distance of 3.833 $\AA$ along the $a$-axis. Thus the $s y n$ head-head dimer obtained is the direct consequence of this packing arrangement. It is noteworthy that coumarin which does not undergo dimerization in its stable modification (5) in the solid state undergoes a clean topochemical photoreaction when substituted by an acetoxy group. Thus it is evident that the acetyl group plays a strategic role in steering coumarin rings to pack themselves into a $\beta$ stacked structure. Earlier, interaction involving overlap of an ester group of one molecule with a benzene ring of another had been utilized to steer diacrylic acids into structures suited for solid state polymerization (4). However, perusal of the figure reveals no such interaction in our case.

Photostability of some of the coumarins (Table 1) in the solid state is not due to any inherent electronic or steric properties of these molecules as they all dimerize readily in solution. It must be attributed to unfavourable packing of these molecules in the crystal lattices. The results of our study on 7-acetoxycoumarin reveal that electronic and steric aspects play, if any, only a minor role in the solid state reactions. It is significant that 7-acetoxycoumarin does not dimerize in solution (7), probably owing to the deactivation of the active 

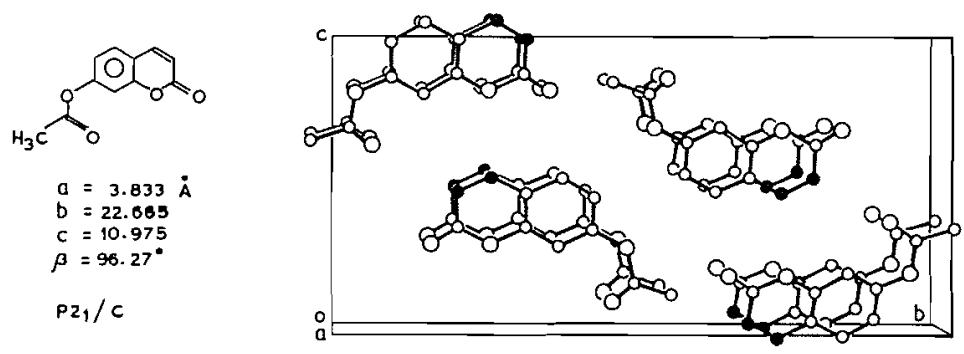

$\beta$-TYPE PACKING OF 7-ACETOXYCOUMARIN DOWN a AXIS

FIG. 1.

double bond by the electron withdrawing acetoxy group. Therefore, it appears that adoption of a crystal packing arrangement favourable for bimolecular reaction can overcome the disadvantageous inherent electronic factors. Attempts are underway to explore the generality of acetoxy group effects in "crystal engineering".

\section{Acknowledgements}

The University Grants Commission and Council of Scientific and Industrial Research, Government of India are thanked for financial assistance. We are grateful to Dr. S. Chandrasekaran for mass spectra.

1. H. Morrison, H. Curtis, and T. McDowell. J. Am. Chem. Soc. 88, 5415 (1966); R. Hof Fman, P. Wells, and H. Morrison. J. Org. Chem. 36, 102 (1971).
2. G. M. J. SCHMIDT et al. Solid state photochemistry. Edited - by D. Ginsburg. Verlag Chemie. 1976; J. M. ThOMAs, S. E. Morsi, and J. P. Desvergne. Ady. Phys. Org. Chem. 15, 63 (1977).

3. G. M. J. SchmidT. Pure Appl. Chem. 27, 647 (1971)

4. H. Nakanishi, W. Jones, J. M. Thomas, M. Hasegawa, and W. L. ReEs. Proc. R. Soc. London Ser. A, 369, 307 (1980).

5. J. Bergman, K. Osaki, G. M. J. Schmidt, and F. I. Sonntag. J. Chem. Soc. 2021 (1964).

6. N. Ramasubbu, T. N. Guru Row, K. Venkatesan, V. Ramamurthy, and C. N. R. Rao. J. Chem. Soc. Chem. Commun. 178 (1982).

7. L. H. Leenders, E. Schouteden, and F. C. De SCHR YVER. J. Org. Chem. 33, 957 (1973).

8. G. M. J. SCHMIDT. J. Chem. Soc. 2014 (1964)

9. P. Main, S. J. Fiske, S. E. Hull, L. Lessinger, G. Germain, J. P. DeclercQ, and M. M. Woolfson. MULTAN-80. System of Computer Programmes, University of York, York, England. 1980.

\title{
On cardioactive steroids. IX. The conversion of digitoxigenin to bufalin
}

\author{
Thomas Y. R. TSAI AND Karel Wiesner \\ Natural Products Research Centre, University of New Brunswick, Bag Service \#45222, Fredericton, N.B., Canada E3B 6 E2 \\ Received May 20, 1982
}

Thomas Y. R. TSAI and Karel Wiesner. Can. J. Chem. 60, 2161 (1982).

A conversion of digitoxigenin I to bufalin 7 via a novel furan-to-pyrone transformation is described. It is hoped that this process will be useful in a simple and efficient total synthesis of bufalin, as well as various other synthetic problems.

Thomas Y. R. TSai et Karel Wiesner. Can. J. Chem. 60, 2161 (1982).

On décrit une transformation de la digitoxigénine (I) en bufaline (7) par l'intermédiaire d'une nouvelle transformation du furanne en pyrone. On espère que ce procédé sera utile dans le cas d'une synthèse simple et efficace de la bufaline et également dans d'autres synthèses.

[Traduit par le journal]

A few years ago we reported a novel and very efficient methodology for the synthesis of cardenolides featuring furan-containing intermediates $(1,2)$. In order to use this methodology for the synthesis of bufadienolides, an equally efficient furan-topyrone transformation is required. We have worked out recently (using a model system) four different transformations of this type $(3,4)$. In the 